\title{
Optimal service order for mass-casualty incident response.
}

Behrooz Kamali (kamali@vt.edu)*, ${ }^{*}$, Douglas Bish (drb1@vt.edu) ${ }^{\dagger}$, Roger Glick (reglick@carilionclinic.org) $\ddagger$

†Grado Department of Industrial and Systems Engineering (0118)

250 Durham Hall Virginia Tech, Blacksburg, VA

$\ddagger$ Carilion Roanoke Memorial Hospital, Roanoke, VA 24014

* Corresponding author

(C) 2017. This manuscript version is made available under the Elsevier user license http://www.elsevier.com/open-access/userlicense/1.0/ 


\begin{abstract}
In the aftermath of a mass-casualty incident, one of the first steps in the response is to triage the casualties. Triage systems categorize the casualties based on criticality, and then prioritize casualties for transfer to hospitals for further treatment. The prioritization is usually based on simply ordering the casualty types without considering the available resources to transport them and the scale of the disaster. These factors can significantly affect the outcome of the rescue efforts. In this research we study a mathematical model to incorporate the above mentioned factors in the triage process. We assume a disaster location with a set of casualties, categorized by criticality and care requirements, that must be transported to hospitals in the region using a fleet of available ambulances. The goal is to maximize the expected number of survivors. We analyze the structure of the optimal solution to this problem, and compare the performance of the model with the current practice and other related models in the literature.
\end{abstract}

Keywords: OR in disaster relief; Emergency management; Mass-casualty triage; Service order

\title{
1 Introduction
}

In this paper we study the response to mass-casualty incidents (MCI). Unlike other emergencies, MCIs are of sufficient scale that the decision-maker (i.e., the incident commander) must manage the response under limited resources (e.g., medical transport). Thus, planning and management are critical to allocate limited resources as efficiently as possible (Antommaria et al., 2011). After an MCI, the first responders initially gather and triage the casualties. Triage is the process of categorizing casualties based on the severity of their injuries, and is an essential MCI management tool; its purpose is to use the available resources in the most efficient manner.

There are several triage methods for MCIs, including START (Simple Triage and Rapid Treatment Method), Homebush, Triage Sieve, Sacco Triage Method (STM), and CESIRA (Lerner et al. 2008) (hospital emergency departments have their own triage methods, see Iserson and Moskop, 2007, for details). For most triage methods there is an implied service order for transporting casualties to hospitals, which is based on the triage categories and does not account for the available resources (e.g., ambulances), the scale of the incident, or the casualty mix. For instance, START, one of the most common triage method in the US (Cone and MacMillan, 2005), has four colorcoded categories: immediate (red) for the most critical casualties that need attention within an hour, delayed (yellow) for serious injuries, but not expected to deteriorate for several hours, minor (green) for victims with relatively minor injuries, and expectant (black) for victims unlikely to survive (Benson et al., 1996). START has an implied service order of most critical to least critical (i.e., red, yellow, and then green), regardless of the situation. A review of the literature reveals that there has been no scientific evaluation of effectiveness of START or any of the other common triage methods (Jenkins et al., 2008; Lerner et al., 2008). One measure of triage effectiveness is the survival rate among the casualties that are treatable (e.g., those in the red, yellow, and green START categories) in the hours to days following the incident. The research question we are studying is "how can we find a service order that maximizes the survival rate?" This research question was motivated in part by our participation in a Full Scale Exercise (FSE) conducted by a regional airport, which simulated a plane crash with a large number of casualties (played by moulaged volunteers). The simulated response included the triage and transport of casualties to nearby hospitals. The triage method used was START. Of the nearby hospitals, one is designated as a Level 1 Trauma Center (the highest level of treatment), which is best able to effectively treat the more critical casualties, while the other hospitals are well suited for less critical casualties.

While we will discuss the literature that address this service order problem later, one interesting facet is when casualty deaths are assumed to occur. Sacco et al. (2005); Mills et al. (2011, 2013); 
Dean and Nair (2014) assume casualty deaths occur after service (e.g., at the hospital), and the survival probability decreases as service is delayed. Conversely, Argon et al. (2008, 2011); Jacobson et al. (2012) study the service order problem under the assumption that casualty deaths occur before service, using a Markovian model and assuming that lifetimes and service times are exponentially distributed (no previous study has shown that exponential distribution is a well-suited distribution for lifetime or service time in an emergency response effort). Based on our reading of the medical and emergency management literature, casualty deaths are more likely to occur after service (as modeled in Sacco et al., 2005, Mills et al., 2011, 2013, Dean and Nair, 2014) rather than before service (as modeled in Argon et al., 2008, 2011; Jacobson et al., 2012), at least for the relevant casualty types (i.e., not expectant in START). For instance, Frykberg and Tepas III (1988) studied 220 terrorist bombing incidents with 2,934 casualties surviving the attack and waiting for service. They recorded 40 subsequent deaths among the 2,934 survivors, of which only one occurred prior to receiving service. Sacco et al. (2005) generate survival probabilities for different casualty types (based on the STM triage system) from historical trauma data, which is used in a model that assumes casualty deaths occur after service.

Li and Glazebrook (2011) study the service order problem considering imperfect classification of casualties using a Bayesian approach in dealing with uncertainties and develop heuristics approaches for the problem. There are two types of misclassification: assignment of a more-critical casualty to a less-critical type, undertriage, and assignment of a less-critical casualty to a more-critical type, overtriage. Frykberg (2002) state that undertriage could lead to preventable deaths and it should be avoided in both daily and mass-casualty triage operations, but state no reported case of undertriage exists in the past bombing attacks. In dealing with daily trauma patients, overtriage occurs at a rate of around $50 \%$ (Kreis Jr et al., 1988), which can also be necessary to avoid preventable deaths by reducing undertriage as much as possible (Frykberg, 2002). On the other hand, Frykberg and Tepas III (1988) conclude overtriage could be as deadly as undertriage in a mass-casualty incident. Overtriage of less-critical casualties could delay service for more-critical casualties and potentially threaten their survival. In contrast, Hupert et al. (2007) find that overtriage could have mixed effects on outcome of triage operation through simulation analysis. In this paper, we study the optimal triage service order problem under the more realistic assumption that casualties do not die before service (but they do deteriorate, thus decreasing their survival probability); triage misclassification is beyond the scope of this paper.

Currently, triage decisions are made based on fixed rules, regardless of the available resources and the number or mix of casualties. The main contributions of this paper can be summarized as follows: 1) we develop a tractable model that determines the optimal service order considering the mix of casualties, casualty type dependent service times, multiple servers, and more than two casualty types; 2 ) we compare our model with others in the literature, considering both structural properties and performance; 3) we identify structural properties of the optimal solution, extending and generalizing the work in other papers; and, 4) we discuss important data issues, and more realistic problems, including multiple servers and casualty types.

The remainder of this paper is organized as follows. In $\S 2$, we describe the problem and provide our model formulation. We also analyze some of the characteristics of the model. Moreover, we provide formulations for other models from the literature and analyze their assumptions and characteristics. In $\S 3$, we introduce the survival data that we use in this paper and discuss it in detail. We study the problem under a simplified case with two casualty types and multiple servers in $\S 4$, and the general case with more than two casualty types in $\S 5$. Finally, conclusions and future works are discussed in $\S 6$. 


\section{Model Description}

Consider a mass casualty incident (MCI). First responders triage the casualties and place them into one of the casualty types in the ordered set $P$, where type 1 is the most critical and type $|P|$ the least critical. For instance, in START type 1 would be immediate casualties (identified with red), type 2 delayed casualties (identified with yellow), and type 3 for casualties with minor injuries (green). The number of casualties of type $i \in P$ are denoted by $n_{i}$. We assume that all casualties are available for service at time zero. There are $N_{t}$ servers (e.g., an ambulance and crew) available at time $t$ (this parameter usually increases over time as more resources arrive from surrounding areas), which work in a non-preemptive manner. Service times, $s_{i}, i \in P$, include any care required to stabilize the casualty and the round trip travel time to an appropriate hospital. Because travel time represents a significant part of the service time, higher criticality does not necessarily imply longer service times. In the motivating FSE (see $\S 1$ ) the hospital that is designated as a level 1 Trauma Center (which represents the highest level of trauma care), would be more appropriate for the more critical casualties, while other hospitals in the vicinity would be appropriate for less critical casualties. Thus the travel times (and thus service times) depend on the location of the MCI, in relation to the various hospitals and there abilities. The objective is to determine a service order that maximizes the number of survivors, given survival probability functions $f_{i t}$ for each casualty type $i$ when service is delayed until time $t$. (See, for instance, Sacco et al., 2005, Mills et al. 2013, which provides survival curves derived from data.).

First, we introduce Model 1, an integer programming formulation for the service order problem. The definitions of the sets and parameters, decision variables, and formulation follow. To model this problem we divide the time horizon into $T$ time intervals, and assume the data is discrete.

\section{Sets and Parameters:}

$P \quad$ set of casualty types in the triage system

$n_{i} \quad$ number of casualties of type $i$ at the beginning of time interval $1, \forall i \in P$

$s_{i} \quad$ number of time intervals it takes to serve a casualty of type $i, \forall i \in P$

$T$ number of time intervals required to serve all the casualties, $\sum_{i \in P} n_{i} s_{i}$

$f_{i t}$ survival probability for a casualty of type $i$ that is served in time interval $t, \forall i \in P$, $t=1, \ldots, T$

$N_{t}$ number of servers available at time interval $t, t=1, \ldots, T$

\section{Decision Variable:}

$x_{i t}$ number of casualties of type $i$, whose service starts at time $t, \forall i \in P, t=1, \ldots, T$ 


$$
\begin{aligned}
\text { Model 1: } \max & \sum_{i \in P} \sum_{t=1}^{T} f_{i t} x_{i t} \\
\text { s.t. } & \sum_{t=1}^{T} x_{i t}=n_{i}, \quad \forall i \in P \\
& \sum_{i \in P} \sum_{f=1}^{\min \left(s_{i}, t\right)} x_{i(t-f+1)} \leq N_{t}, \quad t=1, \ldots, T \\
& x_{i t} \geq 0 \text { and integer, } \forall i \in P, t=1, \ldots, T
\end{aligned}
$$

Objective function (1) maximizes the sum of the survival probabilities of all casualties at the time of service. Survival probability for each casualty is observed at the time of service, as we assume emergency personnel will perform required interventions to stabilize the casualty when the service begins. Thus, the decrease in survival probability rate during the service period is not considered. Constraint (2) guarantees that all the casualties are served. Constraint (3) limits the number of active servers to the number of servers available. Constraint (4) is the integer and non-negativity constraint.

Proposition 1. For Model 1 with a continuous relaxation of the $x$-variables, there exists an optimal solution in which the $x$-variables have integer values.

Proof. The coefficient matrix $A$, has $|P|+T$ rows and $T|P|$ columns. We show that $A$ is totally unimodular and use this to prove the proposition. Order the columns (variables) of $A$ by casualtytype from 1 to $|P|$, and within each type, by time intervals from 1 to $T$. The rows are ordered Constraint (2) from 1 to $|P|$, and then Constraint (3) from 1 to $T$. This yields an $A$ matrix like the one in Figure 1, where in sub-matrix $i \in P$, all $T$ elements in row $i$ are 1 and rest of the rows are all 0 , while for rows $i \in\{|P|+1, \ldots,|P|+T\}$ all elements are zero, except for $\max \left\{1, i-|P|-s_{i}+1\right\}, \ldots, i-|P|$, which are 1 (in the figure, non-zero coefficients are displayed, everything else is zero, and we set $s_{1}=2, s_{2}=3$, and $s_{|P|}=4$, arbitrarily for illustration purposes). Given this form of $A$, we perform the following basic matrix operations. For each sub-matrix $i \in P$ we subtract column $j=T, \cdots, 2$ from columns $k=j-1, \cdots, \max \left\{0, j-s_{i}+1\right\}$. Next we multiply the first $|P|$ rows by -1 . The ordering and column operations used do not affect total unimodularity (Schrijver, 1998), and now the $A$ matrix is such that each column has at most two non-zero values, one +1 and one -1 , which indicates that $A$ is totally unimodular. Given that the right-hand-side values are integer, by Cramer's Rule, all $x$ decision variables will be integer-valued at each extreme point (Bazaraa et al., 2011), and the LP relaxation will have an integer valued optimal solution.

Next, we present other models from the literature using our notation (for easier comparison), these models all represent special cases of Model 1 for more narrowly defined problems. Sacco et al. 2005, 2007) present a linear program for the triage service order problem: 


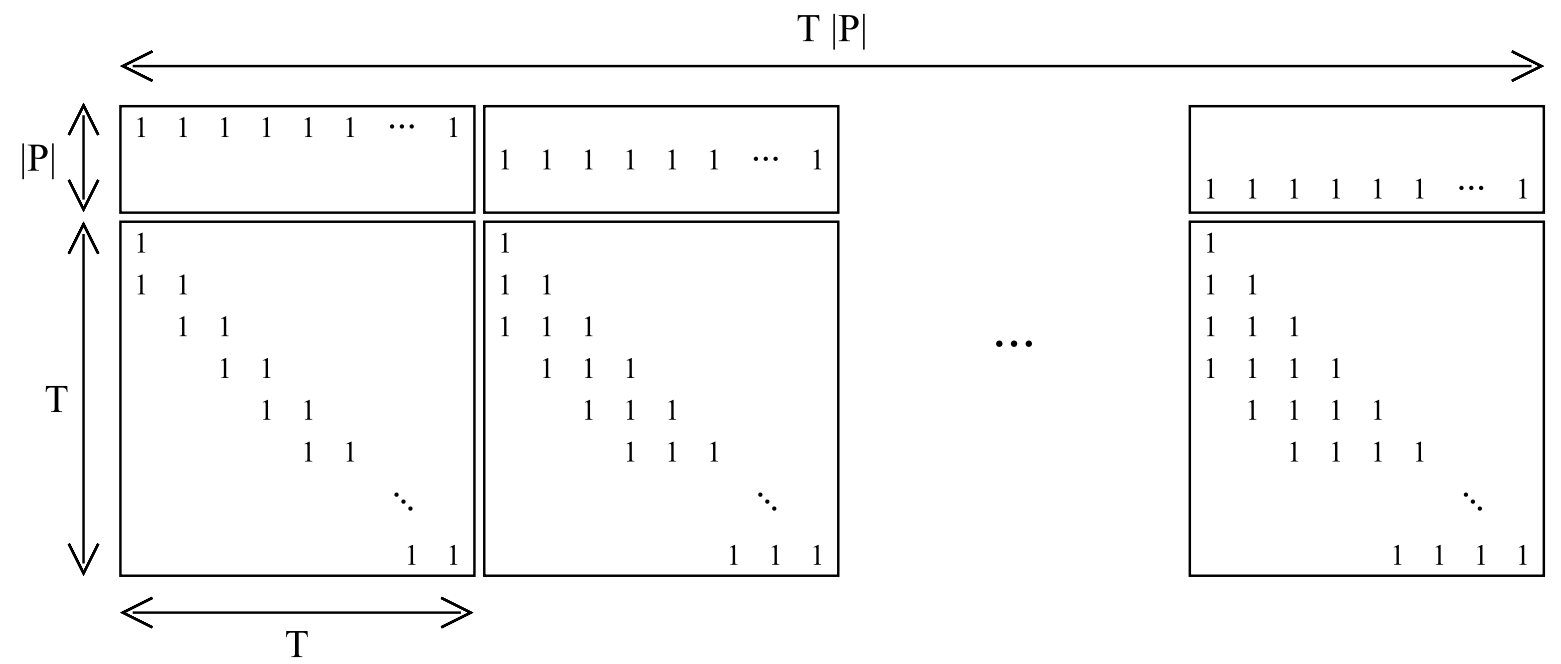

Figure 1: Structure of the coefficients matrix, $A$, for Model 1

$$
\begin{aligned}
\text { Model 2: } \max & \sum_{i \in P} \sum_{t=1}^{T} f_{i t} x_{i t} \\
\text { s.t. } & \sum_{t=1}^{T} x_{i t}=n_{i}, \quad \forall i \in P \\
& \sum_{i \in P} x_{i t} \leq N_{t}, \quad t=1, \ldots, T \\
& x_{i t} \geq 0, \quad \forall i \in P, t=1, \ldots, T .
\end{aligned}
$$

Objective function (5) is identical to (1), while Constraint (6) ensures that all the casualties are served. Constraint (7) limits the number of servers to those available, and Constraint (8) is the integer and the logical non-negativity constraint. This LP is similar to Model 1, except that it implicitly assumes service times are equal for each casualty type, which is a limitation. By Proposition 1, this LP has integer optimal solutions, much like Model 1.

Next, is Model 3A, proposed by Mills et al. (2013), to study the service order problem with one server. Model 3A also assumes that service times for all casualty types are equal, and for simplicity sets them all to one time interval (e.g., service time is the transportation time from the disaster location to the receiving hospital), thus $T=\sum_{i \in P} n_{i}$.

$$
\begin{aligned}
\text { Model 3A: } \max _{W} & \sum_{i \in P} \int_{W(i)} f_{i}(t) d t \\
\text { s.t. } \quad & \mu(W(i))=n_{i}, \quad i \in P \\
& \bigcup_{i \in P} W(i)=[0, T], \\
& W(i) \cap W(j)=\emptyset, \quad \forall i, j \in P, i \neq j,
\end{aligned}
$$


where $W$ is a set-valued decision variable such that $W=\{W(i), i \in P\}$, and $W(i)$ identifies continuous time intervals in which service is allocated to casualty type $i \in P$. The objective function (9) maximizes the sum of survival probabilities, $f_{i}(t), i \in P$, for all casualty types. Constraint (10) guarantees that total number of the casualties served over the time horizon, $\mu(W(i)), i \in P$, is equal to total number of casualties in each category, $n_{i}, i \in P$. Constraints (11) and (12) ensure that service times for all casualty types do not exceed the time horizon $T$ or overlap, respectively. Model 3A, while not a linear program, is equivalent to Model 2 restricted to a single server. Mills et al. (2013) show that there exists an optimal solution to Model 3A where only one casualty type is served at any given time. This result follows from Proposition 1, which shows that there exists an integral optimal solution for the continuous relaxation of the problem, and restricting the problem to a single server. While Model 3A allows for an arbitrary number of casualty types, Mills et al. (2013) simplifies Model 3A for a special case having only two casualty types (thus $T=n_{1}+n_{2}$ ) to gain insight into the structure of the optimal solution. Furthermore, the use of two casualty types is of practical interest as it represents the two casualty types from START that are most significant to the problem (i.e., immediate and delayed). This simplification, denoted as Model 3B, uses an alternate objective function based on the function $g(t)=f_{2}(t)-f_{1}(t)$.

$$
\begin{array}{ccc}
\text { Model 3B: } & \max & \int_{W(2)} g(t) d t+C \\
& \text { s.t. } & W(2)=n_{2}, \\
& W(2) \subseteq[0, T] .
\end{array}
$$

Objective function (13) maximizes the survival probability for two casualty types, here $C$ is a constant defined as $C \equiv \int_{0}^{T} f_{1}(t) d t$, which is equivalent to 9 for two casualty types. The constant is for the survival probability of type 1 casualties, to which we add the difference function $g(t)$. Constraint (14) ensures all type 2 casualties are served. Since the time horizon is limited to exactly the required service time for all casualties $\left(T=n_{1}+n_{2}\right)$, after the assignment of type 2 casualties, the remaining time intervals are assigned to type 1 casualties. Constraint (15) limits type 2 casualties service to the defined time horizon. In this model, each service requires one time interval, independent of casualty type, therefore we can easily discretize the difference function $g(t)$ for each time interval, that is, $g_{t}=f_{2 t}-f_{1 t}$, which we sum for every interval a casualty of type 2 is served, which is indicated by the binary variable $x_{t}\left(x_{t}=1\right.$ if a casualty of type 2 is served in interval $t$, else $x_{t}=0$ and a type 1 casualty is served), which yields Model 3C (once again $\left.T=n_{1}+n_{2}\right)$.

$$
\begin{aligned}
\text { Model 3C: } & \max \\
& \sum_{t=1}^{T} g_{t} x_{t} \\
\text { s.t. } & \sum_{t=1}^{T} x_{t}=n_{2} \\
& x_{t} \in\{0,1\}, \forall t \in 1, \cdots, T .
\end{aligned}
$$

Objective function (16) maximizes the survival probability, and is equivalent to objective function (13) in Model 3B. Constraint (17) ensures all type 2 casualties are served, and it is equivalent to Constraint (14). Finally, Constraint (18) limits the service to the time horizon, which is equivalent to Constraint (15). 
Models 1 through $3 \mathrm{C}$ have a hierarchical relationship; Model 1 is the most general, while Models 3B and $\mathrm{C}$ are for the most narrowly defined problem. Model 1 can address multiple casualty types, a time varying number of servers, and a unique service time for each casualty type. Unlike Model 1, Model 2 requires equal service times for all casualty types, while Model 3A is equivalent to Model 2, but limited to a single server. Model 3B is a simplification of Model 3A with two casualty types, and Models 3B and 3C are equivalent. Table 1 summarizes the differences among all the models.

\begin{tabular}{l|lllll}
\hline Feature & Model 1 & Model 2 & Model 3A & Model 3B & Model 3C \\
\hline Casualty types & Unlimited & Unlimited & Unlimited & Two & Two \\
Servers & Multiple & Multiple & Single & Single & Single \\
Service times & Variable & Equal & Equal & Equal & Equal \\
\hline
\end{tabular}

Table 1: Comparison of service order models

In addition, Dean and Nair (2014) develop an integer programming model similar to Model 1, but with the additional decision of hospital assignment based on the availability of a reusable, casualty-type specific, hospital resources (e.g., beds). Due to the added complexity of their model it is more difficult to solve and to develop insight into the structure of the optimal solution.

Addressing multiple casualty types is important, as there are often casualties that do not fit neatly into the two START classifications discussed above. For instance, pediatric casualties benefit from specialized care and services that should be considered in the management of an MCI. In fact, pediatric casualties have their own triage system, as well as hospitals that specialize in children, and children often represent a significant number of the MCI casualties (Lyle et al., 2009; Mace and Bern, 2007). Because each casualty type has different service and care requirements, the expected service times vary among different casualty types and models should be able to address this difference in order to generate practical results. Another issue ignored by the models in the literature is how number of servers could vary through time during response efforts. It is likely that there are a few servers at the disaster location initially, but their numbers could potentially be augmented through time as part of the regional response. Model 1 captures all these essential elements of an MCI response. Despite this, we first look at the problem with two casualty types, to study the structural solution properties of this problem.

\section{Survival Data}

In this section we discuss the survival probability functions used in the numerical analysis, which are based on the scaled log-logistic survival functions derived in Mills et al. (2013) from data in Sacco et al. (2005) and discuss data issues. The survival probability function is as follows:

$$
f_{i t}=\frac{\beta_{0, i}}{\left(t / \beta_{1, i}\right)^{\beta_{2, i}}+1}, \quad \forall i \in\{1,2\},
$$

where $\beta_{0, i}$, scales the function to the initial survival probability for casualty type $i$, and $\beta_{1, i}$ and $\beta_{2, i}$ are parameters of the log-logistic distribution. Sacco et al. (2005) derive survival probabilities for a 13 category triage system using a retrospective analysis of data from 76,459 casualties from trauma centers in Pennsylvania, where category 0 has the lowest survival probability and 12 the highest. Then, using a Delphi technique, the transition from less critical to more critical categories through time were estimated. Mills et al. (2013) produce five scenarios where the 13 casualty categories from Sacco et al. (2005) are probabilistically mapped into the two START categories of 
interest (see Mills et al., 2013, for details); while Sacco et al. (2005) assume a transition through the casualty types due to delay in service, Mills et al. (2013) have a survival probability function for each casualty type, and no transitioning of casualties between types. Scenario 1 is the most time-sensitive (lower survival probabilities) and Scenario 5 is the least time-sensitive.

\begin{tabular}{c|ccc|ccc}
\hline & \multicolumn{3}{|c}{ Type 1 } & \multicolumn{3}{c}{ Type 2 } \\
Scenario & $\beta_{0,1}$ & $\beta_{1,1}$ & $\beta_{2,1}$ & $\beta_{0,2}$ & $\beta_{1,2}$ & $\beta_{2,2}$ \\
\hline 1 & 0.09 & 17 & 1.01 & 0.57 & 61 & 2.03 \\
2 & 0.15 & 28 & 1.38 & 0.65 & 86 & 2.11 \\
3 & 0.24 & 47 & 1.30 & 0.76 & 138 & 2.17 \\
4 & 0.40 & 59 & 1.47 & 0.77 & 140 & 2.29 \\
5 & 0.56 & 91 & 1.58 & 0.81 & 160 & 2.41 \\
\hline
\end{tabular}

Table 2: Parameters for five survival probability scenarios from Mills et al. (2013) for survival probability function 19 for 5-minute time intervals

Much of the analysis and heuristic development in Mills et al. (2013) is based on the following assumption on the relationship between the two survival probability functions, $f_{i t}, i \in 1,2$.

Assumption 1. There exists a time $t_{m}$ such that $f_{1 t}^{\prime}<f_{2 t}^{\prime}$ for $t<t_{m}, f_{1 t_{m}}^{\prime}=f_{2 t_{m}}^{\prime}$, and $f_{1 t}^{\prime}>f_{2 t}^{\prime}$ for $t>t_{m}$.

Assumption 1 indicates that, before time $t_{m}$, the survival probability of type 1 casualties, $f_{1 t}$, initially decreases at a faster pace than the survival probability of type 2 casualties, but after time $t_{m}$, the type 2 survival probability starts to decrease faster than the survival probability of type 1. Mills et al. (2013) state that the survival probability functions defined in (19) using the data from Table 2 adhere to Assumption 1. This data is for 5-minute time intervals, and throughout this paper we transform survival probability function (19) to account for this.

These five scenarios are problematic in two ways. First, consider these scenarios from the triage perspective. Triage methods place casualties into types based on a (simple) algorithm. For example, based on the START algorithm, where classification is based on pulse rate, respiratory rate, and responsiveness of victims (Benson et al., 1996), there is no way for the first responder to know which survival probability curves are applicable. Under Scenario 1 type 2 casualties have an initial survival probability of 0.57 and an expected lifetime (here lifetime represents the time after which service will not result in survival, which of course is not observable at the time of service) of 269.1 minutes, while under Scenario 5 type 1 casualties have an initial survival probability of 0.56 and an expected lifetime of 554.3 minutes. Thus, the Scenario 1 type 2 casualties, while having a slightly higher initial survival probability than the Scenario 5 type 1 casualties, are the more time critical, i.e., their survival probability drops faster (thus the lower expected lifetime). Also, the initial survival probabilities for type 1 casualties range from 0.09 to 0.56 . Thus, we observe a wide variance between casualties of the same type in different scenarios, and a large overlap in criticality between the two casualty types in the different scenarios. Given that the survival probability curves are crucial in determining the optimal response, any modeling effort would require more stable survival probability curves for each casualty type. These scenarios were produced by probabilistically mapping the 13 casualty types (based on a more complex algorithm) from Sacco et al. (2005) into two casualty types (see Mills et al., 2013, for details). This does not necessarily reflect the START algorithm, but the stability of any triage algorithm is beyond the scope of this paper.

The other issue with the five scenarios is the shape of the survival probability curves, specifically as defined by the initial survival probability. For example, consider Scenario 2. For a type 1 
casualties the initial survival probability is $\beta_{0,1}=0.15$, which results is a flattened log-logistic survival probability curve having an expected lifetime of 62.8 minutes, that represents casualties that having a low survival probability, but are not very time-critical (the low expected lifetime somewhat reflects low initial survival probability, i.e., $85 \%$ of casualties will eventually die no matter how fast service is provided, and the remaining $15 \%$ have a longer life expectancy). The type 2 casualties have a higher probability of survival (initially 0.65), and an expected lifetime of 417.6 minutes. This increases the relative time criticality of the type 2 casualties as their survival probability has much more opportunity to drop.

Relating these two issues and again considering START, should casualties that have, at best, a $9 \%$ or $15 \%$ chance of survival be classified as immediate (i.e., red, and given the highest priority) or expectant (i.e., black, and given the lowest)? Thus, we have identified two areas for further study, first is the quality of the triage algorithm, for instance, if the START algorithm does produce varying scenarios like those described in Scenarios 1-5, then a more accurate algorithm is required, one that better categorizes casualties. We also question the scaling values in Scenarios 1-5 (i.e., the initial survival probability), which, if too low, makes giving type 1 casualties higher priority less desirable. We do not argue that the proposed survival probability data and function proposed by Mills et al. (2013) and Sacco et al. (2005) are either a good or a bad fit, and in this paper we use the five scenarios for comparison purposes.

\section{Multiple Servers and Two Casualty Types}

In this section, we study the structure of the optimal solution to the triage service order problem with two casualty types and multiple servers. Considering only two casualty types helps us show analytical results, and is somewhat practical as in most common triage methods, there are typically two critical casualty types competing for resources. Again, for START these two types are immediate and delayed. The minor category can wait longer before receiving service, while the expectant category has a very low survival probability, regardless of availability of the resources. We extend the work in Mills et al. (2013) by considering multiple servers and more importantly, potentially unequal service times for the two casualty types, and provide alternative solution algorithms to this more general problem. In our analysis, we consider two simple strategies, a strategy where all type 1 casualties are served first, followed by the type 2 casualties, which we denote as $S(1,2)$; this is the implied service order of the START triage system, and we also consider the opposite order, which we denote as $S(2,1)$.

Assumption 1 uses derivatives to identify $t_{m}$. Instead it is more appropriate to use the change in survival probability over the service time (which is more accurate, and important for unequal service times). Given this, we define the following parameter for the change in survival probability:

Definition 1. $f_{i t}^{\delta} \equiv f_{i t}-f_{i[t+\delta]}$.

$f_{i t}^{\delta}$ denotes the decrease in survival probability starting at time $t$ over the next $\delta>0$ time intervals. Given this definition, we make the following assumption:

Assumption 2. There exists a time $t_{s}$ such that $f_{1 t}^{s_{2}}>f_{2 t}^{s_{1}}$ for all $t<t_{s}, f_{1 t_{s}}^{s_{2}}=f_{2 t_{s}}^{s_{1}}$, and $f_{1 t}^{s_{2}}<f_{2 t}^{s_{1}}$ for all $t>t_{s}$.

Assumption 2 is a generalization of Assumption 1 that allows for unequal service times for two casualty types, and survival functions that adheres to the latter, also adhere to Assumption 2, and vise versa. When a type 1 casualty is being served, service to a type 2 casualty is delayed by $s_{1}$ 
time intervals (i.e., until service to type 1 is finished). Thus, decrease in type 2 survival probability is $f_{2 t}^{s_{1}}$, and using the same logic, decrease in survival probability for type 1 is $f_{1 t}^{s_{2}}$.

When service times are equal $\left(s_{1}=s_{2}\right)$, Mills et al. (2013) shows that for one server $(m=1)$ and survival probabilities that adhere to Assumption 2, there exists an optimal solution structured such that service of type 2 casualties form a continuous interval which allows optimal solutions to be described using three time intervals, the first interval for serving $n_{1}^{\prime}, 0 \leq n_{1}^{\prime} \leq n_{1}$ type 1 casualties, the second for serving all $n_{2}$ casualties, and third intervals for serving the remaining $n_{1}-n_{1}^{\prime}$ type 1 casualties. Using this optimal structure, Mills et al. (2013) proposes two heuristics for Model 3B (equal service times and one server). One heuristic, denoted QS-ReSTART, simply decides on either $S(1,2)$ or $S(2,1)$ as the service order plan, while the second heuristic, denoted QD-ReSTART, provides a plan that is either $S(1,2), S(2,1)$, or time-dependent, which serves type 1 casualties until the estimated switching point, $\tau$, after which service switches from type 1 to type 2. If $\tau \leq 0$, QD-ReSTART results in $S(2,1)$, if $\tau \geq n_{1} s_{1}$ it results in $S(1,2)$.

Next, we introduce Algorithm 1 that produces optimal solutions when service times are equal $\left(s_{1}=s_{2}\right)$, even considering $m$ servers and survival probabilities that do not necessarily adhere to Assumption 2. To describe this greedy knapsack algorithm, we assume, without loss of generality, that service times are one unit. Start with a list ORDER of $n_{1}+n_{2}$ items, where each item has four elements: decision epoch (service time, $t$ ), server, $g_{t}=f_{2 t}-f_{1 t}$, and casualty type. ORDER $[i]_{j}$ refers to the element $j$ of item $i$ in ORDER, for all $i \in\left\{1, \ldots, n_{1}+n_{2}\right\}$ and $j \in\{1,2,3,4\}$. The first for-loop sets all elements of ORDER with the decision epochs, the servers, the $g_{t}$ value for the epoch, and sets the casualty type to 1 (for type 1). Next, ORDER is sorted on $g_{t}$ (third element) from largest to smallest. Finally, the first $n_{2}$ items, those having the highest $g_{t}$-values, are re-set to type 2 casualties, that is, the fourth element in ORDER is set to 2. ORDER now indicates the optimal service order and server for each casualty.

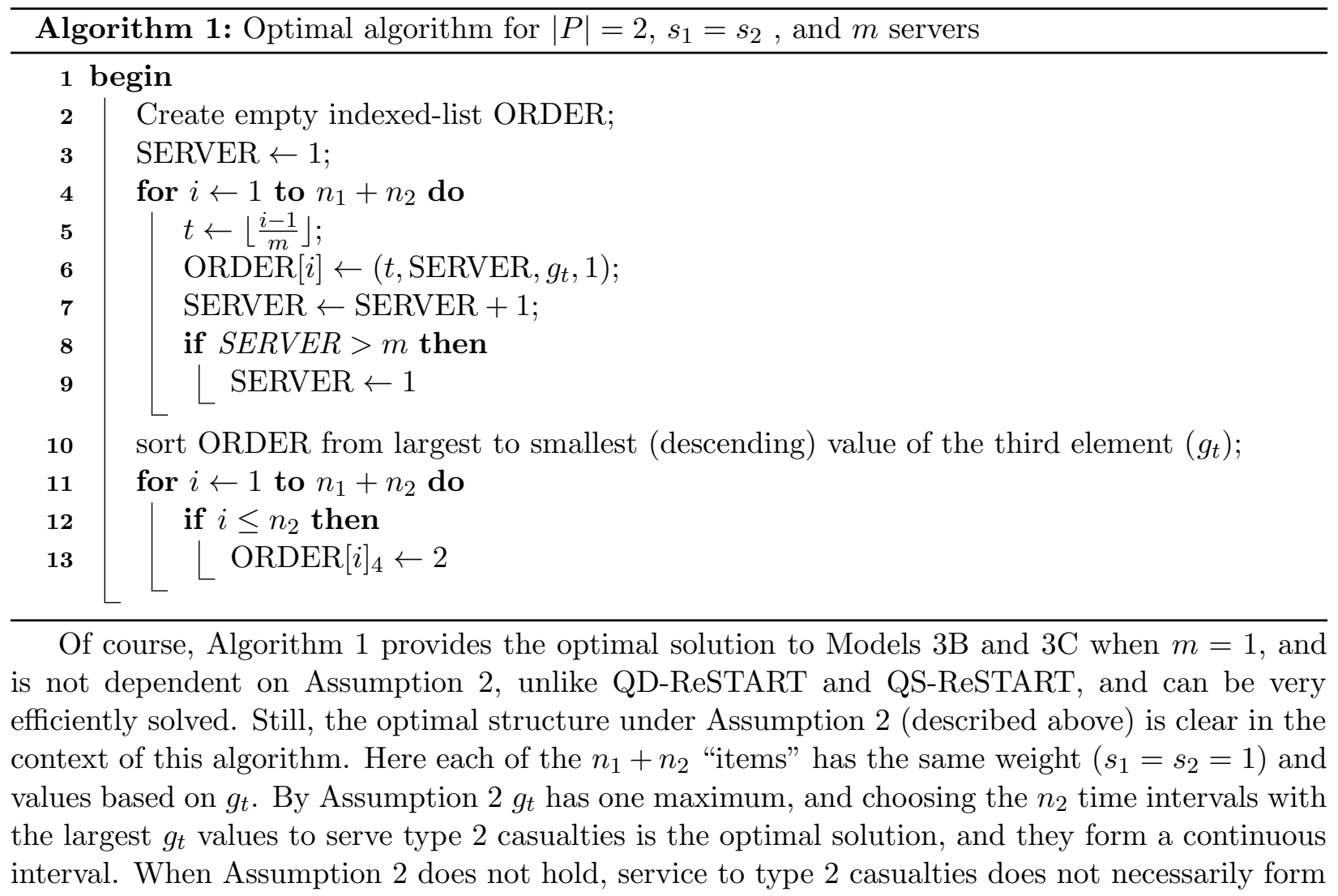


a continuous interval.

Next, we consider unequal service times and multiple servers. In Mills et al. (2013) the rational for equal service times is that they are based on the time required to transport a casualty to the hospital. But often there are multiple hospitals to consider, and sending all the casualties to the closest hospital is often not a good idea, instead this decision should be based on the capabilities of each of the regional hospitals (e.g., a Level 1 Trauma center is best able to handle more critical patients). Thus, the location of the MCI and the corresponding hospitals would determine the service times. Also, the time required for stabilization of different casualty type could vary, which also leads to unequal service times. We now offer a generalization of the structural results from Mills et al. (2013).

Proposition 2. There exists an optimal solution to Model 1 under Assumption 2, where service of type 2 casualties once started, is not interrupted in any of the servers.

Proof. See Appendix.

Proposition 2 shows that with unequal service times, the optimal solution for each server has a similar structure to that with equal service times, and that service is balanced across the servers. We serve type 2 casualties in an interval containing $t_{s}$, assuming there are sufficient casualties. Increasing the number of servers does not change $t_{s}$, but it does change $t_{i}^{*}$ (the time for server $i$ to switch from type 1 to type 2 casualties). As the number of servers increases, $t_{i}^{*}$ either remains the same, or increases. Having multiple servers favors the $S(1,2)$ solution. Consider the interval in which type 2 casualties are being served (depending on $t_{s}$ ) when there is one server. Adding another server reduces this interval (still around $t_{s}$, assuming sufficient number of casualties), potentially increasing the length of the first interval serving type 1 casualties, and of course more type 1 casualties will be served in this first interval because of the increased number of servers, which we see in the results displayed in Table 3 , from Model $1, S(1,2)$, and $S(2,1)$ strategies for two casualty types and different number of servers. Base on the same logic, as we add more servers, more type 1 casualties are served initially (assuming there is a sufficient number of them), as $t_{s}$ remains the same and type 2 service gets more condensed around that point. If there are insufficient number of type 1 casualties, we finish service to them and start serving type 2 casualties earlier. In this case, the optimal service order becomes $S(1,2)$. This confirms favoring $S(1,2)$ as we increase number of the servers.

Unlike the special case where service times are equal, this is not a simple knapsack problem. There is not a well-defined set of decision epochs, rather the time of decision epochs depends on previous decisions, and thus survival probabilities in the next decision epoch depend on the casualty type that is currently being served. Despite this, if the survival probability function adheres to Assumption 2, we know the optimal structure, which allows us to use a simple search, starting with the $S(2,1)$ strategy, evaluating the expected number of survivors, and then moving one type 1 casualty to the end of the service queue on each server and again evaluate the expected number of survivors, and repeat until the expected number of survivors decreases. Algorithm 2 shows the detailed steps to find the optimal solution having two casualty types and $m$ servers, with no restriction on service times. Building on the insight from the Proposition 2 , Algorithm 2 generates the optimal service order by finding the point in which service should be switched from type 1 to type 2 . The algorithm starts with $S(2,1)$ strategy on each server, while number of the casualties are balanced on each server (equal or almost equal number of type 1 and type 2 casualties on each server). The solution is then presented in terms of three service intervals on server $j, n_{1, j}^{\prime}$ type 1 , $n_{2, j}$ type 2 , and $n_{1, j}-n_{1, j}^{\prime}$ type 1 . If $n_{1, j}^{\prime}=n_{1}$ for all $j \in\{1, \ldots, m\}$, solution is the same as $S(1,2)$ 
and if $n_{1, j}^{\prime}=0$ for all $j \in\{1, \ldots, m\}$, the solution is $S(2,1)$.

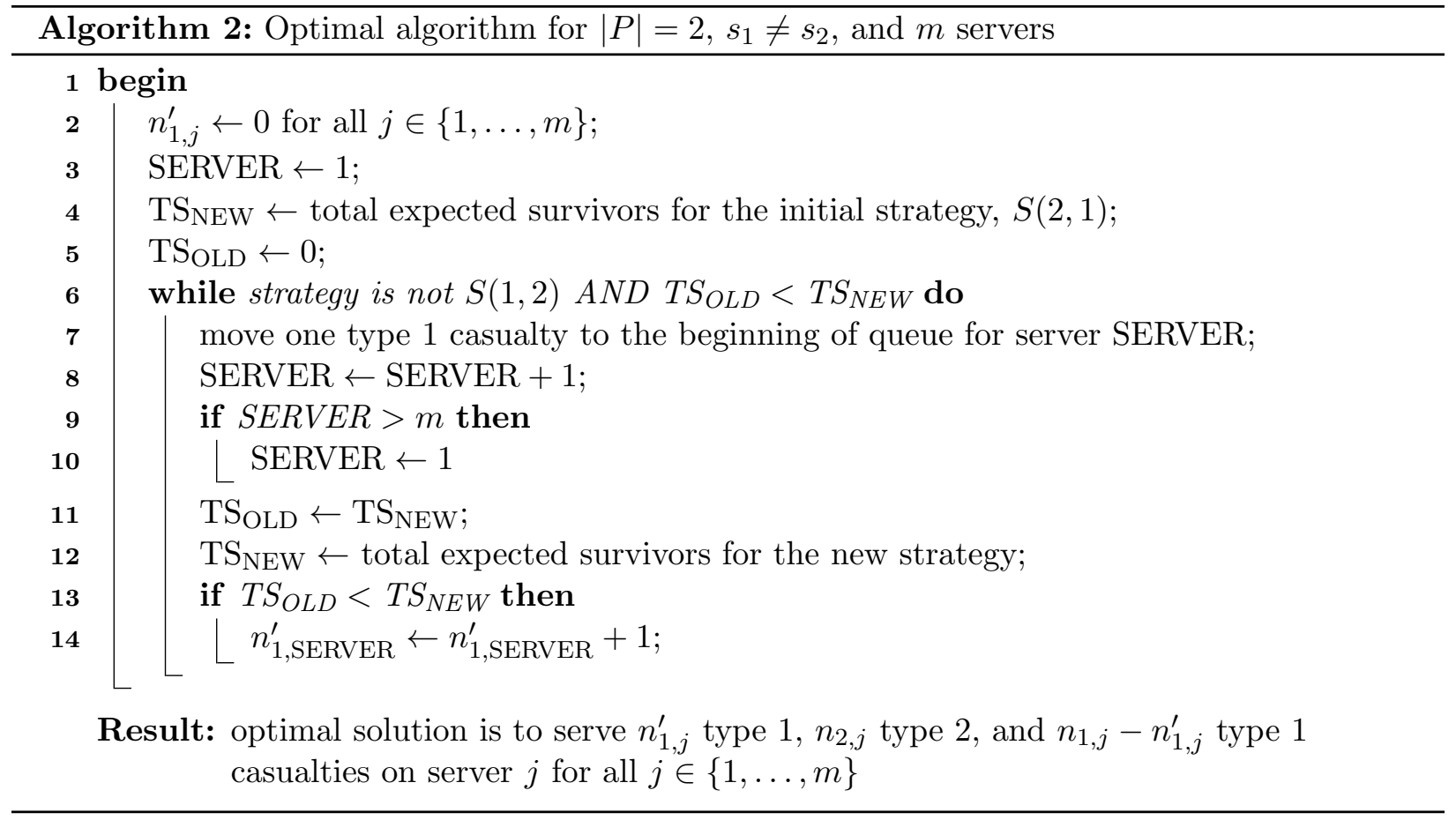

Figure 2 displays $f_{i t}^{s}$ for Scenario 5 (see Table 2 ) where $s=s 1=s 2=30$ minutes. The dashed curve (red) is the change in type 1 survival probability function over the 30 minute service time and the solid curve (yellow) is for type 2 casualties. As expected, both functions are decreasing with type 1 initially decreasing at a faster pace until point $t_{s}=341$ (see Assumption 2), after which the type 2 function decreases faster. Given sufficient number of casualties, the interval in which type 2 casualties are served contains $t_{s}$ in the optimal solution. For example, in Figure 2, the optimal solution for six cases, which vary in the mix of casualties, is illustrated. For each of these cases, a bar represents the service decisions, the white bars (with red outline) indicates service to type 1 casualties and solid (yellow) section indicates service to type 2. In each case, there are total of 50 casualties and number of the casualties served in each interval is stated on the graph. The graph illustrates that as we increase number of type 2 casualties, their service interval grows around $t_{s}$ and their service is not interrupted.

Figure 3 illustrates optimal solutions from Model 1 when service times are not equal $\left(\left(s_{1}, s_{2}\right)=\right.$ $(35,25)$, both in minutes) under Scenario 5 , these solutions adhere to Proposition 2. The dashed curve (red) is $f_{1 t}^{s_{2}}$, the change in survival probability for type 1 casualties per service time of casualty type 2, and solid curve (yellow) is $f_{2 t}^{s_{1}}$ for casualty type 2. As in Figure 2, white bars (with red outline) indicate service of type 1 casualties, and the solid intervals (yellow) indicate service of type 2 casualties. Comparing Figures 2 and 3 , we observe increasing type 1 service and decreasing type 2 service times decrease $t_{s}$, which for the given case under Scenario 5 decreased the $t_{s}$ from 341 to 268 , and moves the results towards $S(2,1)$, as it becomes less efficient to serve type 1 casualties with greater service time and lower survival probability using limited resources compared to the type 2 casualties with shorter service time and higher survival probability.

Earlier in Assumption 2 we showed that $t_{s}$ is dependent on the service times. Thus, we cannot estimate the results from multiple servers by proportionally reducing service times. Based on the proposition, we can do so by proportionally reducing the number of the casualties (This does not 


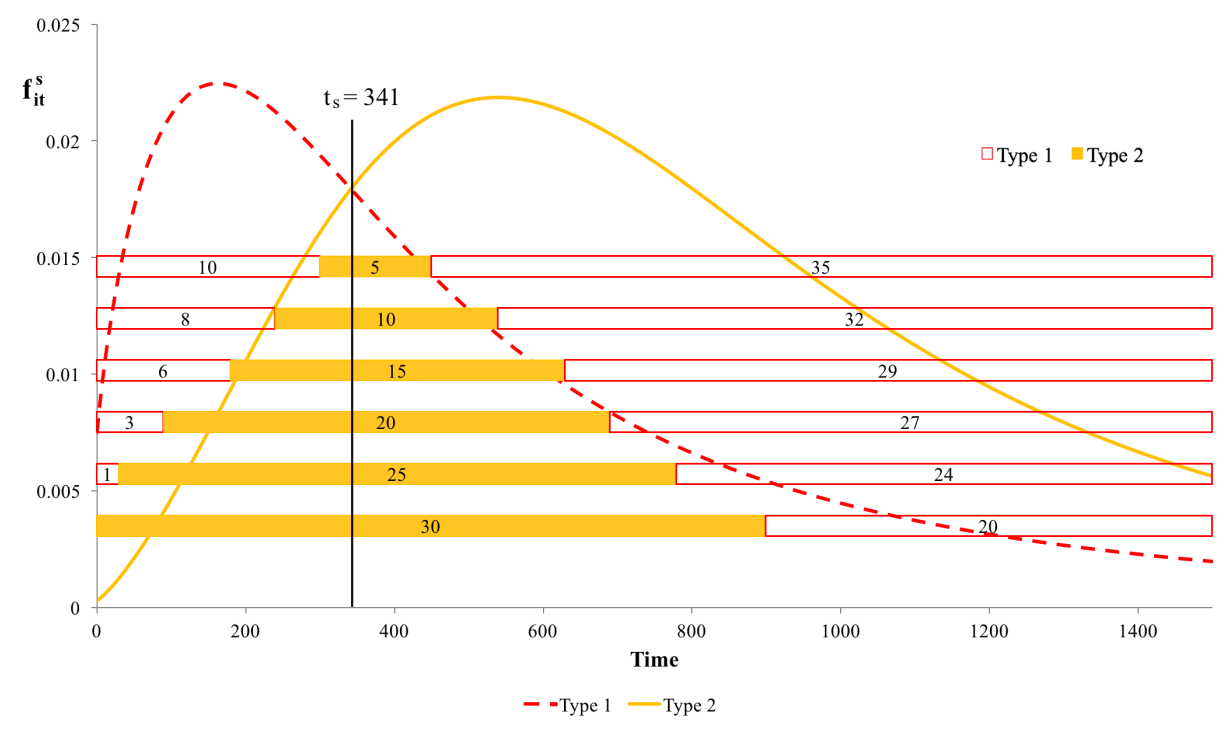

Figure 2: Optimal service order for six different mixes of casualties with $s=s 1=s 2=30$ for Scenario 5

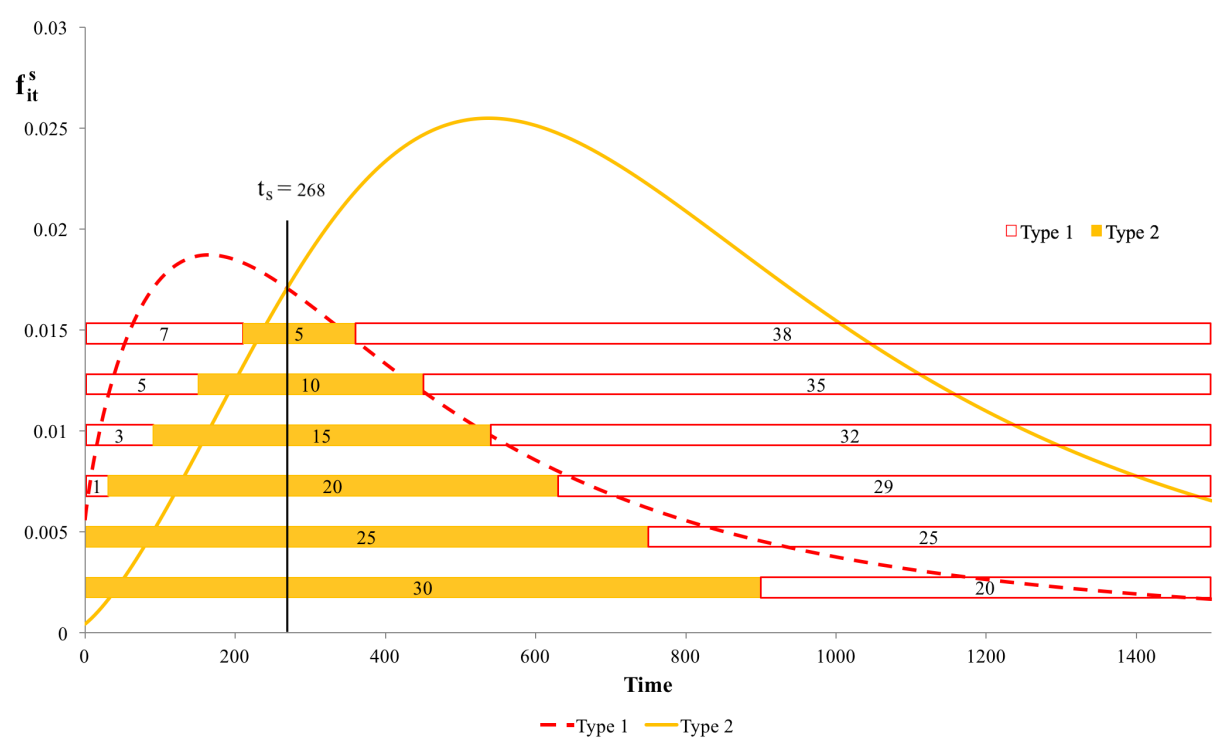

Figure 3: Optimal service order for different mix of casualties with $s_{1}=35$ and $s_{2}=25$ for Scenario 5

provide the optimal solution, but provides a more accurate estimation of the optimal solution). Mills et al. (2013) approximate the solution with multiple servers by dividing service times by number of the servers, but this can cause errors as the number of servers increase. Consider Scenario 5 with $n_{1}=25$ and $n_{2}=25$ and 5 servers, each having a service time of 30 minutes. The optimal solution serves five type 1 casualties, then 25 type 2 casualties, and then the remaining 20 type 1 casualties. For this solution the expected number of survivors is 4.845 type 1, 15.804 type 2 , for a total of 20.649. The approximation is obtained using one server with a service time of 6 minutes. This has an optimal solution that serves two type 1 casualties, then 25 type 2 , followed by the remaining 23 type 2 casualties. For this solution the expected number of survivors is 3.454 type 1, 16.198 type 
2, for a total of 19.652 survivors. This approximate method has an error of 0.997 in the number of expected survivors in this example. In addition, as stated in Assumption 2, reducing service times change $t_{s}$, which results in a different switching time $t^{*}$ that changes the optimal solution (based on Proposition 2).

Table 3 displays numerical results for cases with one, two, and three servers from Model 1, $S(1,2)$, and $S(2,1)$ strategies using the survival probabilities generated from 19$)$. Each scenario is simulated 5,000 times using service times for each casualty of type $i$ that are lognormally distributed with mean $s_{i}$ and standard deviation $0.4 s_{i}$ (as done in the Mills et al. (2013) simulation analysis and justified from an empirical study by Ingolfsson et al. (2008) as a good fit for ambulance travel times). The mean service time $\left(s_{i}\right)$ is uniformly distributed between 20 to 40 minutes, and randomly generated for each of the 5,000 instances. For each instance, the randomly generated $s_{i}$ is used as a deterministic parameter in Model 1 . Thus in most cases service times for the casualty types are not equal. All instances have 50 casualties, the number of type 1 casualties is uniformly distributed between 10 and 40, and the remaining casualties are type 2. We report the number of times each method generates the policy having the highest number of survivors (reported as Best in the table), as well as the the average number of the casualties of type 1, type 2, and total, that survive across the 5,000 instances. Note, because of the stochasticity and the discrete nature of the objective function (counting the number of survivors), two different policies, e.g., mixed and $\mathrm{S}(1,2)$, can both be best for any particular instance.

\begin{tabular}{|c|c|c|c|c|c|c|c|c|c|c|c|c|}
\hline \multirow[b]{3}{*}{ Method } & \multicolumn{4}{|c|}{1 Server } & \multicolumn{4}{|c|}{2 Servers } & \multicolumn{4}{|c|}{3 Servers } \\
\hline & \multirow[b]{2}{*}{ Best } & \multicolumn{3}{|c|}{ Average Survived } & \multirow[b]{2}{*}{ Best } & \multicolumn{3}{|c|}{ Average Survived } & \multirow[b]{2}{*}{ Best } & \multicolumn{3}{|c|}{ Average Survived } \\
\hline & & Type 1 & Type 2 & Total & & Type 1 & Type 2 & Total & & Type 1 & Type 2 & Total \\
\hline & \multicolumn{12}{|c|}{ Scenario 1} \\
\hline Model 1 & 4,824 & 0.17 & 6.55 & 6.73 & 4,639 & 0.35 & 9.98 & 10.33 & 4,538 & 0.49 & 11.27 & 11.76 \\
\hline $\mathrm{S}(1,2)$ & 66 & 0.54 & 1.33 & 1.88 & 249 & 0.88 & 4.33 & 5.21 & 381 & 1.03 & 6.28 & 7.31 \\
\hline \multirow[t]{2}{*}{$\mathrm{S}(2,1)$} & 4,779 & 0.17 & 6.54 & 6.72 & 4,598 & 0.34 & 9.97 & 10.32 & 4,536 & 0.49 & 11.28 & 11.76 \\
\hline & \multicolumn{12}{|c|}{ Scenario 2} \\
\hline Model 1 & 4,789 & 0.24 & 9.31 & 9.55 & 4,361 & 0.75 & 12.83 & 13.58 & 3,994 & 1.18 & 13.96 & 15.14 \\
\hline $\mathrm{S}(1,2)$ & 70 & 1.09 & 2.58 & 3.67 & 518 & 1.80 & 7.04 & 8.84 & 909 & 2.16 & 9.62 & 11.77 \\
\hline \multirow[t]{2}{*}{$\mathrm{S}(2,1)$} & 4,765 & 0.24 & 9.31 & 9.55 & 4,363 & 0.63 & 12.94 & 13.56 & 3,960 & 0.93 & 14.16 & 15.09 \\
\hline & \multicolumn{12}{|c|}{ Scenario 3} \\
\hline Model 1 & 4,682 & 0.86 & 13.83 & 14.68 & 3,986 & 2.33 & 16.72 & 19.04 & 3,816 & 3.31 & 17.33 & 20.64 \\
\hline $\mathrm{S}(1,2)$ & 108 & 2.44 & 5.84 & 8.28 & 1,013 & 3.59 & 12.16 & 15.75 & 1,855 & 4.12 & 14.66 & 18.78 \\
\hline \multirow[t]{2}{*}{$\mathrm{S}(2,1)$} & 4,674 & 0.83 & 13.85 & 14.68 & 3,614 & 1.73 & 17.10 & 18.82 & 3,077 & 2.33 & 17.91 & 20.24 \\
\hline & \multicolumn{12}{|c|}{ Scenario 4} \\
\hline Model 1 & 4,242 & 1.76 & 14.00 & 15.76 & 3,575 & 5.01 & 16.47 & 21.48 & 3,520 & 6.51 & 17.32 & 23.83 \\
\hline $\mathrm{S}(1,2)$ & 354 & 4.59 & 5.94 & 10.53 & 1,559 & 6.62 & 12.52 & 19.14 & 2,381 & 7.49 & 15.11 & 22.61 \\
\hline \multirow[t]{2}{*}{$\mathrm{S}(2,1)$} & 4,110 & 1.49 & 14.25 & 15.74 & 2,643 & 3.27 & 17.47 & 20.74 & 2,068 & 4.43 & 18.26 & 22.70 \\
\hline & \multicolumn{12}{|c|}{ Scenario 5} \\
\hline Model 1 & 3,636 & 4.26 & 15.01 & 19.28 & 3,385 & 9.27 & 17.38 & 26.65 & 3,474 & 10.94 & 18.39 & 29.33 \\
\hline $\mathrm{S}(1,2)$ & 755 & 8.14 & 7.35 & 15.49 & 2,226 & 10.82 & 14.45 & 25.26 & 2,948 & 11.78 & 16.90 & 28.68 \\
\hline $\mathrm{S}(2,1)$ & 3,331 & 3.13 & 15.97 & 19.10 & 1,995 & 6.44 & 18.89 & 25.33 & 1,640 & 8.20 & 19.56 & 27.76 \\
\hline
\end{tabular}

Table 3: Simulation of the results from Model $1, S(1,2)$, and $S(2,1)$ with different number of servers

We observe from the results in Table 3 that increasing the number of servers leads to an increase in total survivors and moves the optimal solution closer to $S(1,2)$, as shown analytically earlier. In more critical scenarios (e.g., Scenario 1) with fewer servers $S(1,2)$ is very unlikely to be the best service order policy, but shorter randomly generated type 2 lifetimes or type 1 service times, longer randomly generated type 1 lifetimes or type 2 service times, or a combination of all could result in 
$S(1,2)$ being the best solution. For Model 1, if $S(1,2)$ is the optimal solution, increasing servers does not change this, while if $S(2,1)$ is the optimal solution, decreasing servers does not change this.

Next, in Table 4, we perform a similar numerical analysis with more realistic initial survival probabilities (as discussed in $\S 3$ ). In all the scenarios, we use $\left(\beta_{0,1}, \beta_{0,2}\right)=(0.8,0.95)$ with the rest of the parameters the same as in Table 2 . The simulation setting is the same as before.

\begin{tabular}{|c|c|c|c|c|c|c|c|c|c|c|c|c|}
\hline \multirow[b]{3}{*}{ Method } & \multicolumn{4}{|c|}{1 Server } & \multicolumn{4}{|c|}{2 Servers } & \multicolumn{4}{|c|}{3 Servers } \\
\hline & \multirow[b]{2}{*}{ Best } & \multicolumn{3}{|c|}{ Average Survived } & \multirow[b]{2}{*}{ Best } & \multicolumn{3}{|c|}{ Average Survived } & \multirow[b]{2}{*}{ Best } & \multicolumn{3}{|c|}{ Average Survived } \\
\hline & & Type 1 & Type 2 & Total & & Type 1 & Type 2 & Total & & Type 1 & Type 2 & Total \\
\hline & \multicolumn{12}{|c|}{ Scenario 1} \\
\hline Model 1 & 4,110 & 1.89 & 10.56 & 12.45 & 3,631 & 4.85 & 15.44 & 20.29 & 3,491 & 6.95 & 17.21 & 24.16 \\
\hline $\mathrm{S}(1,2)$ & 235 & 4.79 & 2.24 & 7.02 & 771 & 7.61 & 7.17 & 14.78 & 1,232 & 9.15 & 10.65 & 19.80 \\
\hline \multirow[t]{2}{*}{$\mathrm{S}(2,1)$} & 4,086 & 1.57 & 10.87 & 12.44 & 2,958 & 3.06 & 16.62 & 19.67 & 2,387 & 4.13 & 18.93 & 23.05 \\
\hline & \multicolumn{12}{|c|}{ Scenario 2} \\
\hline Model 1 & 3,854 & 2.20 & 12.79 & 15.00 & 3,537 & 6.77 & 16.97 & 23.74 & 3,508 & 9.55 & 18.57 & 28.13 \\
\hline $\mathrm{S}(1,2)$ & 261 & 5.71 & 3.76 & 9.47 & 1,155 & 9.44 & 10.28 & 19.72 & 1,815 & 11.38 & 14.02 & 25.40 \\
\hline \multirow[t]{2}{*}{$\mathrm{S}(2,1)$} & 3,524 & 1.33 & 13.58 & 14.90 & 2,188 & 3.29 & 18.94 & 22.22 & 1,552 & 4.86 & 20.74 & 25.60 \\
\hline & \multicolumn{12}{|c|}{ Scenario 3} \\
\hline Model 1 & 3,573 & 4.40 & 16.02 & 20.42 & 3,451 & 40.83 & 76.43 & 58.69 & 3,524 & 12.68 & 20.60 & 33.28 \\
\hline $\mathrm{S}(1,2)$ & 445 & 8.17 & 7.37 & 15.54 & 1,881 & 12.03 & 15 & 27.22 & 2,563 & 13.76 & 18.37 & 32.13 \\
\hline \multirow[t]{2}{*}{$\mathrm{S}(2,1)$} & 2,910 & 2.68 & 17.41 & 20.09 & 1,527 & 5.68 & 21.36 & 27.03 & 996 & 7.67 & 22.41 & 30.07 \\
\hline & \multicolumn{12}{|c|}{ Scenario 4} \\
\hline Model 1 & 3,573 & 5.05 & 15.92 & 20.97 & 3,428 & 11.43 & 19.16 & 30.59 & 3,433 & 13.94 & 20.68 & 34.61 \\
\hline $\mathrm{S}(1,2)$ & 564 & 9.20 & 7.32 & 16.53 & 2,092 & 13.29 & 15.45 & 28.73 & 2,776 & 15.02 & 18.67 & 33.69 \\
\hline \multirow[t]{2}{*}{$\mathrm{S}(2,1)$} & 2,804 & 2.91 & 17.62 & 20.54 & 1,409 & 6.54 & 21.56 & 28.09 & 999 & 8.85 & 22.56 & 31.41 \\
\hline & \multicolumn{12}{|c|}{ Scenario 5} \\
\hline Model 1 & 3,385 & 7.09 & 16.63 & 23.73 & 3,360 & 13.85 & 19.85 & 33.69 & 3,422 & 15.95 & 21.33 & 37.28 \\
\hline $\mathrm{S}(1,2)$ & 901 & 11.67 & 8.58 & 20.25 & 2,375 & 15.50 & 16.93 & 32.43 & 3,040 & 16.83 & 19.88 & 36.71 \\
\hline $\mathrm{S}(2,1)$ & 2,660 & 4.46 & 18.73 & 23.19 & 1,428 & 9.21 & 22.15 & 31.36 & 1,169 & 11.78 & 22.92 & 34.71 \\
\hline
\end{tabular}

Table 4: Simulation of the results from Model $1, S(1,2)$, and $S(2,1)$, with different number of servers, and revised data

Table 4 demonstrates how increasing initial survival probability increases total expected survivors. As expected, increasing initial survival probability moves the results towards $S(1,2)$. This is mainly due to increase in the value of the $t_{s}$ as explained in Survival Data section (e.g., the case explained in Survival Data). As we increase number of the servers, number of the type 2 casualties served around $t_{s}$ in a single server decreases (since the service spreads across a greater number of servers). Thus, potentially the available time to initially serve type 1 casualties increase and results shift towards $S(1,2)$. In general, the pattern of change in the results remain similar to that Table 3. In few cases, even though the average number of total casualties survived is similar for Model 1 and $S(1,2)$, the breakdown of the results shows the service strategies are different. Consider Scenario 5 with 3 servers, here Model 1 and $S(1,2)$ have similar total number of survivors (although the difference of over half a survivor is significant). Of the 5,000 simulation runs, Model 1 generated the (unique) best solution 1,046 times, $S(1,2) 989$ times, and $S(2,1) 525$ times. For the other runs, at least two of the approaches generated the same best result. Examining the Model 1 solutions, the service policy was $S(1,2)$ 1,820 times, $S(2,1) 241$ times, and mixed 2,967 times. Note, these numbers illustrate that two different policies, e.g., mixed and $S(1,2)$, can both produce the same best result (i.e., the same number of survivors), which as we mentioned earlier, is due to the simulation uncertainty and the discrete nature of each simulation run. Thus, while for this scenario $S(1,2)$ clearly dominates $S(2,1)$, there are occasion where $S(2,1)$ does better. 
Model 1 mainly produces mixed policies, but is able to produce $S(1,2)$ and $S(2,1)$ policies, and mixed policies that more closely resemble either of these extremes, as appropriate based on the input parameters, which is related to Table 5, which displays the average of the gaps between the number of survivors in the best solution and number of survivors from each of the methods for the simulation runs of Table 4 (thus the best solution of a particular run has a gap of zero) as well as the maximum gap from the 5,000 runs. As expected, in all cases Model 1 generates smallest average and maximum gap, and in many cases the gap is noticeably larger for $S(1,2)$ and $S(2,1)$. Again examining Scenario 5 with 3 servers, we see the maximum gap for $S(1,2)$ is 14 , while for Model 1 it is 6 . This is because Model 1 is much more flexible, there instances where $S(2,1)$ is the best policy, and in these instances we would expect the $S(1,2)$ policy to do poorly. As another measure, $S(2,1)$ had a gap of 5 or more 298 times, while Model 1 had a gap of that magnitude only 13 times $(S(2,1)$ had a gap of 5 or more 1337 times). Thus using Model 1 is much more robust than the fixed policies.

\begin{tabular}{|c|c|c|c|c|c|c|}
\hline & \multicolumn{2}{|c|}{1 Server } & \multicolumn{2}{|c|}{2 Servers } & \multicolumn{2}{|c|}{3 Servers } \\
\hline Method & Average & Max & Average & $\operatorname{Max}$ & Average & Max \\
\hline & \multicolumn{6}{|c|}{ Scenario 1} \\
\hline Model 1 & 0.21 & 5 & 0.42 & 7 & 0.49 & 8 \\
\hline $\mathrm{S}(1,2)$ & 5.64 & 18 & 5.93 & 26 & 4.86 & 22 \\
\hline \multirow[t]{2}{*}{$\mathrm{S}(2,1)$} & 0.22 & 6 & 1.04 & 13 & 1.60 & 13 \\
\hline & \multicolumn{6}{|c|}{ Scenario 2} \\
\hline Model 1 & 0.35 & 6 & 0.48 & 6 & 0.47 & 11 \\
\hline $\mathrm{S}(1,2)$ & 5.88 & 19 & 4.50 & 23 & 3.20 & 21 \\
\hline \multirow{2}{*}{$\mathrm{S}(2,1)$} & 0.44 & 6 & 2.01 & 16 & 2.99 & 19 \\
\hline & \multicolumn{6}{|c|}{ Scenario 3} \\
\hline Model 1 & 0.47 & 8 & 0.48 & 7 & 0.42 & 7 \\
\hline $\mathrm{S}(1,2)$ & 5.35 & 17 & 2.61 & 17 & 1.57 & 13 \\
\hline \multirow[t]{2}{*}{$\mathrm{S}(2,1)$} & 0.80 & 9 & 2.80 & 18 & 3.63 & 20 \\
\hline & \multicolumn{6}{|c|}{ Scenario 4} \\
\hline Model 1 & 0.50 & 7 & 0.50 & 9 & 0.46 & 7 \\
\hline $\mathrm{S}(1,2)$ & 4.94 & 17 & 2.35 & 17 & 1.38 & 13 \\
\hline \multirow[t]{2}{*}{$\mathrm{S}(2,1)$} & 0.93 & 10 & 2.99 & 17 & 3.66 & 19 \\
\hline & \multicolumn{6}{|c|}{ Scenario 5} \\
\hline Model 1 & 0.60 & 7 & 0.52 & 7 & 0.45 & 6 \\
\hline $\mathrm{S}(1,2)$ & 4.07 & 17 & 1.78 & 16 & 1.01 & 14 \\
\hline $\mathrm{S}(2,1)$ & 1.13 & 11 & 2.86 & 16 & 3.02 & 17 \\
\hline
\end{tabular}

Table 5: Analysis of the gaps between solutions from Model $1, S(1,2)$, and $S(2,1)$ and best solution, with different number of servers, and revised data

\section{More Than Two Casualty Types}

Restricting our analysis to two casualty types provided insight into the structure of the optimal solution, and has some practical value ads discussed above. But there are often more casualty types, for instance, pediatric casualties have their own triage system, and would benefit from additional casualty types. Furthermore, Sacco et al. (2005) propose a triage system with 13 types. In this section, we expand our analysis beyond two casualty types.

Figure 4 illustrates the survival probability function difference, $f_{i t}^{s}$, for three casualty types. Casualty types in the figure follow the data in Table 2, with type 1 (red dashed curve) using the data from type 1 under Scenario 2, and types 2 (orange solid curve) and 3 (green dotted curve) using the data from types 1 and 2 under Scenario 5, respectively. Service times are 30 minutes 


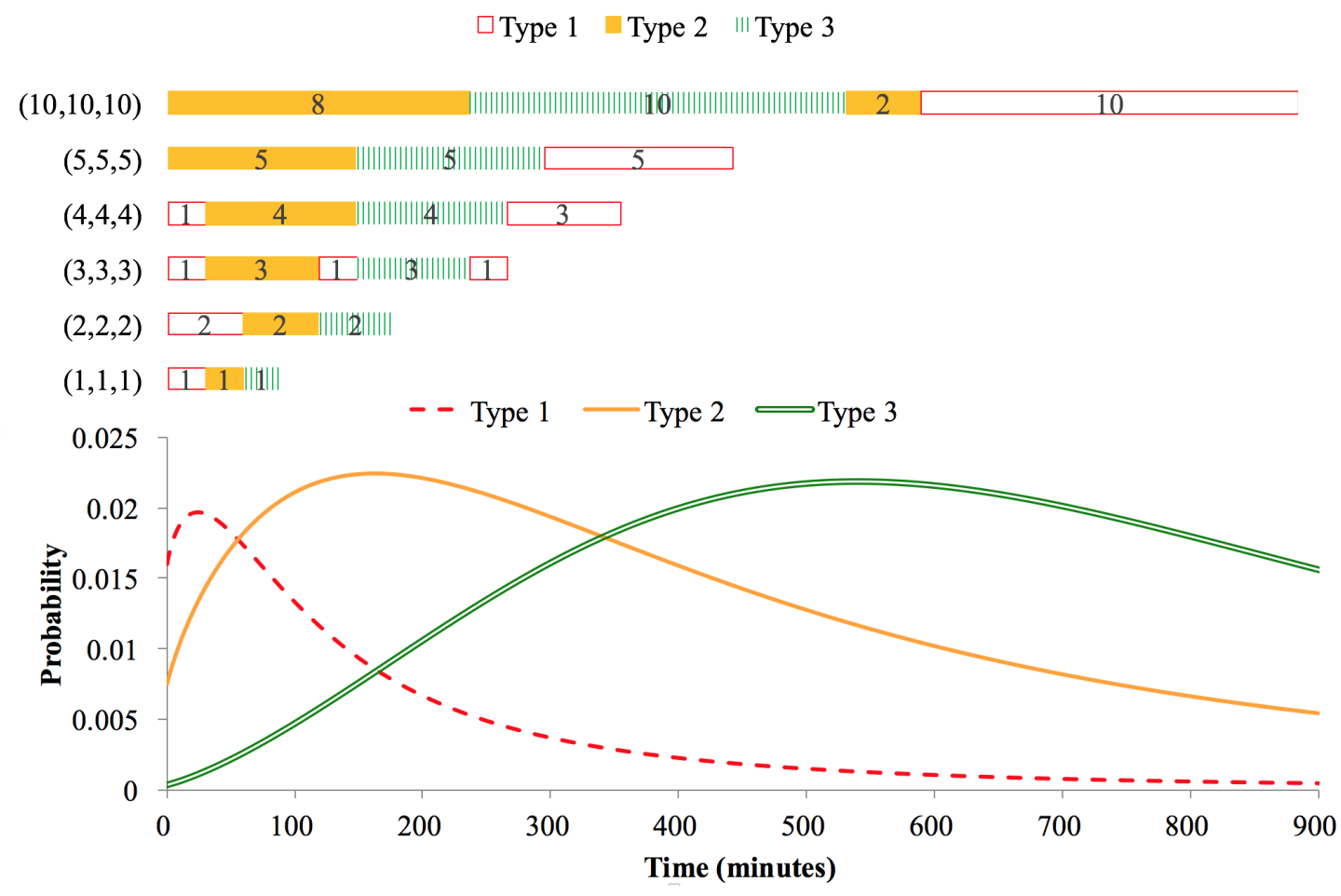

Figure 4: Survival probability difference and optimal solution for three casualty types

for all casualty types. On the top portion of the figure, the optimal solutions for six cases with different number of casualties is depicted. The number of the casualties are shown as $\left(n_{1}, n_{2}, n_{3}\right)$ next to the optimal service priority.

We observe that the structure of the optimal solution follows the pattern identified in Proposition 2 for two casualty types. Starting with $(1,1,1)$ case, the first $t_{s}$ (service times are equal) is at time 55 for curves of type 1 and 2 . Thus, the type 2 casualty is ordered around that point, with type 1 before and type 3 after it. Type 1 casualty is scheduled first as its $f_{1 t}^{s}$ has a larger value than $f_{3 t}^{s}$. For the second case, $(2,2,2)$, we have a similar optimal solution (i.e., $\left.S(1,2,3)\right)$. Two type 2 casualties are scheduled towards the right side of $t_{s}$ of types 1 and 2 curves, as their difference is right-skewed (type 1 curve has a sharp decrease while type 2 has a gradual increase). As we increase number of the casualties, type 2 casualties get scheduled around the related $t_{s}=55$, leaving insufficient room for all type 1 casualties to be served at the beginning. In case $(3,3,3)$, one type 1 is served after type $2 \mathrm{~s}$ and before type $3 \mathrm{~s} . t_{s}=164$ for curves of type 1 and 3 , and type 3 casualties are scheduled around that point in $(3,3,3)$ case leaving room for one type 1 casualty before them. In $(5,5,5)$ case there is no place left for serving type 1 casualties before type 2 or type 3 and service becomes $S(2,3,1)$. Type 3 is the least critical type and its service cannot be interrupted by any of the other types, thus, service to type 1 is pushed to the end. In none of the mentioned five cases type 3 casualties are prioritized over type 2 as their corresponding $t_{s}=341$, and both of these casualty types are served before that point in all cases. As stated in Proposition 2, service of a less critical casualty type is never interrupted by a more critical casualty type. In the last scenario, $(10,10,10)$, as we increase number of the casualty types, scheduling all type 3 casualties around $t_{s}=341$ for curves of types 2 and 3 leaves insufficient room for initial service of all type 2 casualties, and thus, their service is broken into two parts, before type 3 casualties and after. 
We can extend Algorithm 1 to solve the problem for multiple casualty types with equal service times and multiple servers. The extended approach is presented in Algorithm 3 . The first change is introduction of LIST, which is the list of all possible casualty types assignments for every decision epoch and server. Here, instead of a single $g_{t}$ value, we calculate the difference of survival probability curves for a specific casualty type and every other more critical casualty type, and then pick the maximum as the $g_{t}$ for that item. For the most critical casualty type (type 1), this difference is always 0 . After sorting the LIST based on the $g_{t}$ value, we start picking items with the greatest $g_{t}$ values. After each item is picked from LIST, we assign its casualty type to the same decision epoch and server in a results table, ORDER. Following each assignment, all the other items with the same decision epoch and server are removed from the LIST, as no other assignment to the same server at that decision epoch is possible. We continue the same process until there is no other item in LIST. When LIST becomes empty, values in ORDER indicate type of the casualty to be served by the assigned server (corresponding column) at the specified time interval (corresponding row). This algorithm generates an optimal solution, as initially we enumerate all the possible assignments, and then pick the best at each iteration. Also, after each assignment, we only remove the other items for the same epoch and server, thus, each assignment is independent of other assignments for other epochs and servers. The complexity of this algorithm is the same as complexity of sorting the list, as the following steps are only iterating the list linearly. In the algorithm, $\mathrm{EPOCH}_{j}$ refers to the element $j$ of the selected decision epoch, which are time of service $(j=1)$, assigned server $(j=2)$, $g_{t}$ value $(j=3)$, and type of casualty $(j=4)$.

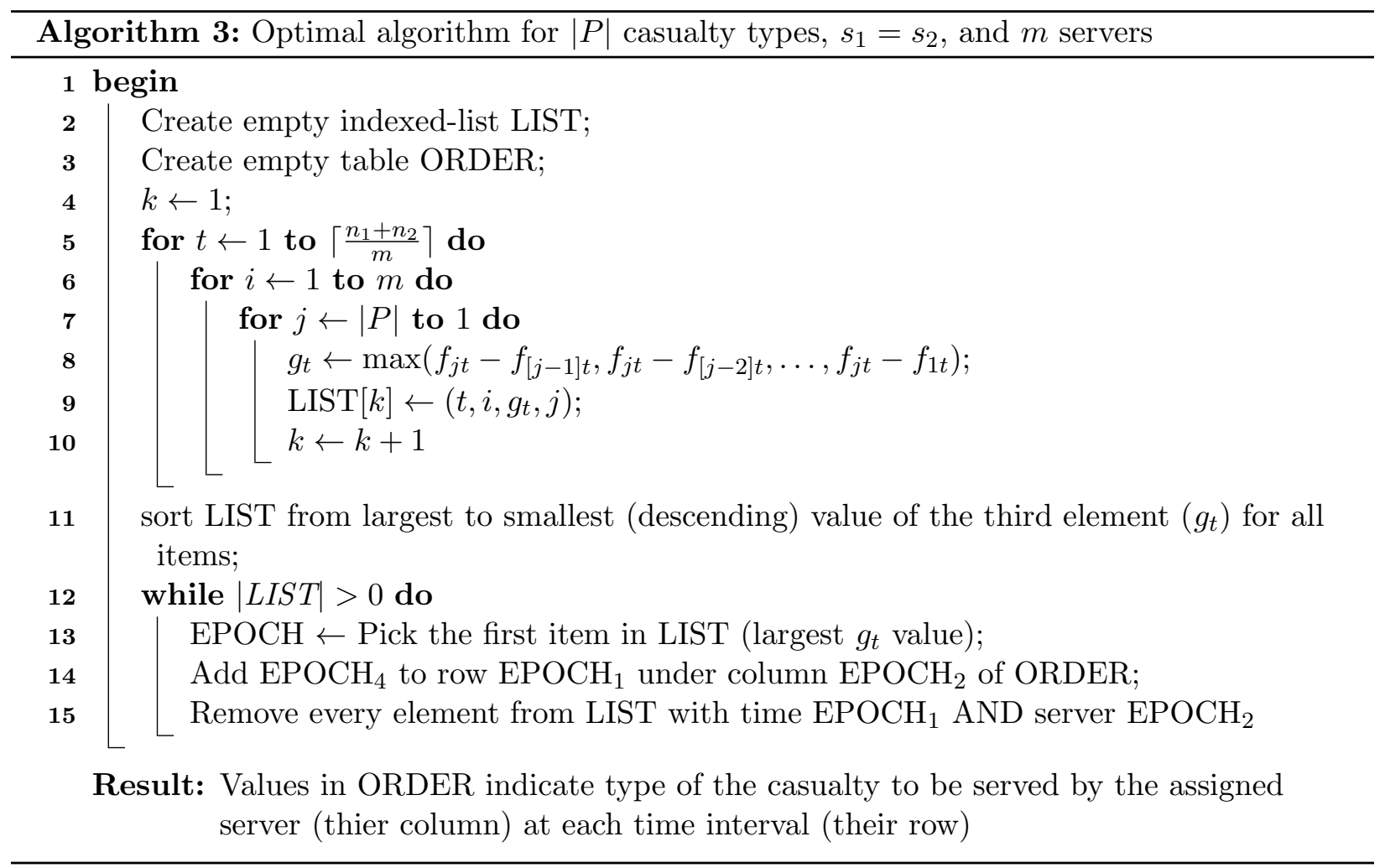

\section{Conclusion and Future Steps}

The medical community has identified the need to incorporate several factors such as scale of the disaster and availability of resources into the triage process. In this paper, we develop a 
mathematical model to show how the scale of the disaster and availability of the resources affects the outcome of the triage operation. Results from the analysis of our model provides decision makers with optimal prioritization policies in order to maximize the expected number of survivors. We achieve this with minimal data requirements, specifically the number of casualties in each category, their service times, and the number of servers available. This information can be collected rapidly to generate the optimal triage plan.

Our findings verify the impact disaster-specific factors can have on the outcome of the triage process. This is in contrast with the current triage processes in practice such as START that have a static prioritization policy regardless of the scale of the disaster and available resources. We show although more critical-first policies similar to $S(1,2)$ might be optimal in some cases, in many other, the reverse or some other mixed strategies perform significantly better. Our analysis allows us to gain some insight into the structure of the optimal policy, which could be the base for future studies and development of easy to implement, but precise policies. The model we present is a generalization of those found in the literature, which allows us to study problems under more practical assumptions, including multiple servers and arbitrary number of casualty types. A further enhancement is that our model readily accepts casualty specific service times, which adds realism. Despite these features, there are still some elements of a response to a mass-casualty indecent that should be considered in the future. We detail some of these in the following paragraphs.

Currently we assume that all casualties are served and there is no death before receiving service. While Frykberg and Tepas III (1988) find that there are small number of deaths among casualties before receiving service, casualty deaths need to be further investigated. We need to study how the possibility of death before receiving service affects the structure of the optimal solution. There has been research for the service order problem under the opposite assumption, namely that deaths occur before service (see Jacobson et al., 2012), but we feel this goes too far.

We also assume that all the casualties are available at the beginning of the relief effort, but in many cases, identification of all casualties might take hours. Stochastic casualty arrival can be incorporated into the model to address this issue and analyze how it affects the optimal policies. Another assumption we made is having one disaster location. In some cases, there are multiple locations hit by a disaster and prioritization and transportation of the casualties should be done simultaneously in multiple locations.

Finally, hospital selection should be considered. Hospitals have limited capacity to treat casualties and different capabilities. These must be considered, else there is the possibility that the hospital becomes overwhelmed, which can lead to a reducing in the ability to treat casualties.

\section{Acknowledgments}

This work was supported in parts by the National Science Foundation (Grant \#1055360) and Carilion Clinic. We thank the reviewers and editors for their valuable contributions. 


\section{References}

Antommaria, A.H.M., Powell, T., Miller, J.E., Christian, M.D., et al. (2011). "Ethical issues in pediatric emergency mass critical care." Pediatric Critical Care Medicine, 12 (6), pp. S163-S168.

Argon, N.T., Ziya, S., and Righter, R. (2008). "Scheduling impatient jobs in a clearing system with insights on patient triage in mass casualty incidents." Probability in the Engineering and Informational Sciences, 22 (3), p. 301.

Argon, N.T., Ziya, S., and Winslow, J.E. (2011). "Triage in the aftermath of mass-casualty incidents." Wiley Encyclopedia of Operations Research and Management Science.

Bazaraa, M.S., Jarvis, J.J., and Sherali, H.D. (2011). Linear programming and network flows. John Wiley \& Sons.

Benson, M., Koenig, K.L., and Schultz, C.H. (1996). "Disaster triage: START, then SAVEa new method of dynamic triage for victims of a catastrophic earthquake." Prehospital and Disaster Medicine, 11 (02), pp. 117-124.

Cone, D.C. and MacMillan, D.S. (2005). "Mass-casualty triage systems: a hint of science." Academic Emergency Medicine, 12 (8), pp. 739-741.

Dean, M.D. and Nair, S.K. (2014). "Mass-casualty triage: Distribution of victims to multiple hospitals using the SAVE model." European Journal of Operational Research, 238 (1), pp. $363-373$.

Frykberg, E.R. (2002). "Medical management of disasters and mass casualties from terrorist bombings: how can we cope?" Journal of Trauma-Injury, Infection, and Critical Care, 53 (2), pp. 201-212.

Frykberg, E.R. and Tepas III, J. (1988). "Terrorist bombings. Lessons learned from Belfast to Beirut." Annals of Surgery, 208 (5), p. 569.

Hupert, N., Hollingsworth, E., and Xiong, W. (2007). "Is overtriage associated with increased mortality? Insights from a simulation model of mass casualty trauma care." Disaster Medicine and Public Health Preparedness, 1 (S1), pp. S14-S24.

Ingolfsson, A., Budge, S., and Erkut, E. (2008). "Optimal ambulance location with random delays and travel times." Health Care Management Science, 11 (3), pp. 262-274.

Iserson, K.V. and Moskop, J.C. (2007). "Triage in medicine, part I: concept, history, and types." Annals of Emergency Medicine, 49 (3), pp. 275-281.

Jacobson, E.U., Argon, N.T., and Ziya, S. (2012). "Priority assignment in emergency response." Operations Research, 60 (4), pp. 813-832.

Jenkins, J.L., McCarthy, M.L., Sauer, L.M., Green, G.B., Stuart, S., Thomas, T.L., and Hsu, E.B. (2008). "Mass-casualty triage: time for an evidence-based approach." Prehospital and Disaster Medicine, 23 (01), pp. 3-8.

Kreis Jr, D.J., Fine, E.G., Gomez, G.A., Eckes, J., Whitwell, E., and Byers, P.M. (1988). "A prospective evaluation of field categorization of trauma patients." Journal of Trauma and Acute Care Surgery, 28 (7), pp. 995-1000. 
Lerner, E.B., Schwartz, R.B., Coule, P.L., Weinstein, E.S., Cone, D.C., Hunt, R.C., Sasser, S.M., Liu, J.M., Nudell, N.G., Wedmore, I.S., et al. (2008). "Mass casualty triage: an evaluation of the data and development of a proposed national guideline." Disaster Medicine and Public Health Preparedness, 2 (Supplement 1), p. S25.

Li, D. and Glazebrook, K.D. (2011). "A Bayesian approach to the triage problem with imperfect classification." European Journal of Operational Research, 215 (1), pp. 169-180.

Lyle, K., Thompson, T., and Graham, J. (2009). "Pediatric mass casualty: triage and planning for the prehospital provider." Clinical Pediatric Emergency Medicine, 10 (3), pp. 173-185.

Mace, S.E. and Bern, A.I. (2007). "Needs assessment: are Disaster Medical Assistance Teams up for the challenge of a pediatric disaster?" The American journal of emergency medicine, 25 (7), pp. $762-769$.

Mills, A., Argon, N.T., and Ziya, S. (2011). "Resource-based START (ReSTART): mass-casualty triage under resource limitations." In Annual Conference of Manufacturing 85 Service Operations Management.

Mills, A.F., Argon, N.T., and Ziya, S. (2013). "Resource-based patient prioritization in masscasualty incidents." Manufacturing \& Service Operations Management.

Sacco, W.J., Navin, D.M., Fiedler, K.E., Waddell, I., Robert, K., Long, W.B., and Buckman Jr, R.F. (2005). "Precise formulation and evidence-based application of resource-constrained triage." Academic Emergency Medicine, 12 (8), pp. 759-770.

Sacco, W.J., Navin, D.M., Waddell, R.K., Fiedler, K.E., Long, W.B., Buckman Jr, R.F., et al. (2007). "A new resource-constrained triage method applied to victims of penetrating injury." The Journal of Trauma and Acute Care Surgery, 63 (2), pp. 316-325.

Schrijver, A. (1998). Theory of linear and integer programming. John Wiley \& Sons. 


\section{Appendix}

Proof of Proposition 2, We divide the proof to two parts; first, we show the proposition holds for one server, and then, we show the works across multiple servers.

PART 1: Without loss of generality, consider a solution to the service order problem, having a partial schedule $S$ starting at time $t^{\prime}$, which specifies serving one type 2 casualty, followed by $m_{1}$ type 1 casualties, and then a second type 2 casualty. We denote the total survival probabilities (i.e., expected number of survivors) generated by the schedule $S$, $T R(S)$, where $T R(S)=f_{2 t^{\prime}}+$ $\sum_{r=0}^{m_{1}-1} f_{1\left[t^{\prime}+s_{2}+r s_{1}\right]}+f_{2\left[t^{\prime}+s_{2}+m_{1} s_{1}\right]}$. Under Assumption 2, we have $f_{1 t}^{s_{2}}>f_{2 t}^{s_{1}}$ for $t<t_{s}, f_{1 t_{s}}^{s_{2}}=f_{2 t_{s}}^{s_{1}}$, and $f_{1 t}^{s_{2}}<f_{2 t}^{s_{1}}$ for $t>t_{s}$. Thus, under Assumption 2 there are three possible cases for where $t_{s}$ lies in regard to schedule $S$, and for each of these we show that schedule $S$, which interrupts the service of type 2 casualties, is suboptimal.

CASE 1: $\left(t_{s}>t^{\prime}+2 s_{2}+m_{1} s_{1}\right.$, thus $\left.f_{1 t}^{s_{2}}>f_{2 t}^{s_{1}}, \forall t=t^{\prime}, \cdots, t^{\prime}+2 s_{2}+m_{1} s_{1}\right)$ : Here we move the first type 2 casualty to the end, to form schedule $S^{\prime}$. Now, we can calculate the difference in the total survival probabilities of schedules $S$ and $S^{\prime}$ as follows:

$$
\begin{aligned}
T R\left(S^{\prime}\right)-T R(S)= & \left(f_{2\left[t+m_{1} s_{1}\right]}-f_{2 t}\right)+\sum_{i=1}^{m_{1}}\left(f_{1\left[t+(i-1) s_{1}\right]}-f_{1\left[t+s_{2}+(i-1) s_{1}\right]}\right) \\
= & \left(f_{2\left[t+m_{1} s_{1}\right]}-f_{2 t}\right)-\sum_{i=1}^{m_{1}}\left(f_{1\left[t+s_{2}+(i-1) s_{1}\right]}-f_{1\left[t+(i-1) s_{1}\right]}\right) \\
= & \left(f_{2\left[t+m_{1} s_{1}\right]}+\sum_{i=1}^{m_{1}-1}\left(f_{2\left[t+i s_{1}\right]}-f_{2\left[t+i s_{1}\right]}\right)-f_{2 t}\right) \\
& -\sum_{i=1}^{m_{1}}\left(f_{1\left[t+s_{2}+(i-1) s_{1}\right]}-f_{1\left[t+(i-1) s_{1}\right]}\right) \\
= & \sum_{i=1}^{m_{1}}\left(f_{2\left[t+i s_{1}\right]}-f_{2\left[t+(i-1) s_{1}\right]}\right)-\sum_{i=1}^{m_{1}}\left(f_{1\left[t+s_{2}+(i-1) s_{1}\right]}-f_{1\left[t+(i-1) s_{1}\right]}\right) \\
T R\left(S^{\prime}\right)-T R(S)= & -\sum_{i=1}^{m_{1}} f_{1\left[t+(i-1) s_{1}\right]}^{s_{2}}+\sum_{i=1}^{m_{1}} f_{2\left[t+(i-1) s_{1}\right]}^{s_{1}}
\end{aligned}
$$

There are $m_{1}$ terms in each summation of the equation (20). We now compare terms in the summations with each other one by one. Given the assumption for this case $\left(f_{1 t}^{s_{2}}>f_{2 t}^{s_{1}}\right)$, we have $f_{1 t}^{s_{2}}>f_{2 t}^{s_{1}}, f_{1\left[t+s_{1}\right]}^{s_{2}}>f_{2\left[t+s_{1}\right]}^{s_{1}}, f_{1\left[t+2 s_{1}\right]}^{s_{2}}>f_{2\left[t+2 s_{1}\right]}^{s_{1}}, \ldots$, and $f_{1\left[t+\left(m_{1}-1\right) s_{1}\right]}^{s_{2}}>f_{2\left[t+\left(m_{1}-1\right) s_{1}\right]}^{s_{1}}$. Thus, we conclude that $\sum_{i=1}^{m_{1}} f_{1\left[t+(i-1) s_{1}\right]}^{s_{2}}>\sum_{i=1}^{m_{1}} f_{2\left[t+(i-1) s_{1}\right]}^{s_{1}}$, and $T R\left(S^{\prime}\right)>T R(S)$. As a result, when $f_{1 t}^{s_{2}}>f_{2 t}^{s_{1}}$, we are always better off serving type 1 casualties first, and then, type 2 casualties.

CASE 2: $\left(t_{s}<t^{\prime}\right.$, thus $\left.f_{1 t}^{s_{2}}<f_{2 t}^{s_{1}}, \forall t=t^{\prime}, \cdots, t^{\prime}+2 s_{2}+m_{1} s_{1}\right)$ : In this case we move the second type 2 casualty to the beginning after the first one, to form schedule $S^{\prime \prime}$. Now, we can calculate the difference in the total survival probabilities of schedules $S$ and $S^{\prime \prime}$ as follows: 


$$
\begin{aligned}
T R\left(S^{\prime \prime}\right)-T R(S)= & \left(f_{2\left[t+s_{2}\right]}-f_{2\left[t+m_{1} s_{1}+s_{2}\right]}\right)+\sum_{i=1}^{m_{1}}\left(f_{1\left[t+2 s_{2}+(i-1) s_{1}\right]}-f_{1\left[t+s_{2}+(i-1) s_{1}\right]}\right) \\
= & \sum_{i=1}^{m_{1}}\left(f_{1\left[t+2 s_{2}+(i-1) s_{1}\right]}-f_{1\left[t+s_{2}+(i-1) s_{1}\right]}\right)-\left(f_{2\left[t+m_{1} s_{1}+s_{2}\right]}-f_{2\left[t+s_{2}\right]}\right) \\
= & \sum_{i=1}^{m_{1}}\left(f_{1\left[t+2 s_{2}+(i-1) s_{1}\right]}-f_{1\left[t+s_{2}+(i-1) s_{1}\right]}\right) \\
= & -\left(f_{2\left[t+m_{1} s_{1}+s_{2}\right]} \sum_{i=1}^{m_{1}-1}\left(f_{2\left[i s_{1}+s_{2}\right]}-f_{2\left[i s_{1}+s_{2}\right]}\right)-f_{2\left[t+s_{2}\right]}\right) \\
& -\sum_{i=1}^{m_{1}}\left(f_{1\left[t+2 s_{2}+(i-1) s_{1}\right]}-f_{1\left[t+s_{2}+(i-1) s_{1}\right]}\right) \\
T R\left(S^{\prime \prime}\right)-T R(S)= & \left.-\sum_{i=1}^{m_{1}} f_{1\left[t+s_{1}+(i-1) s_{1}+s_{2}\right]}^{s_{2}}-f_{2\left[t+(i-1) s_{1}+s_{2}\right]}\right) \\
&
\end{aligned}
$$

Equation (21) has a similar structure to equation (20) and using a similar comparison, we conclude that $T R\left(S^{\prime \prime}\right)>T R(S)$. Therefore, when $f_{2 t}^{s_{1}}>f_{1 t}^{s_{2}}$, we are always better off serving type 2 casualties first, and then, type 1 casualties.

CASE 3: $\left(t^{\prime}<t_{s}<t^{\prime}+2 s_{2}+m_{1} s_{1}\right.$, thus $f_{1 t}^{s_{2}}>f_{2 t}^{s_{1}}, \forall t=t^{\prime}, \cdots, t_{s}$ and $f_{1 t}^{s_{2}}<f_{2 t}^{s_{1}}, \forall t=t_{s}, \cdots, t^{\prime}+$ $\left.2 s_{2}+m_{1} s_{1}\right)$ : To study this case, we break it into two time intervals, $\left[t^{\prime}, t_{s}\right]$ and $\left[t_{s}, t+m_{1} s_{1}+2 s_{2}\right]$. In the first interval we have $f_{2 t}^{s_{1}}<f_{1 t}^{s_{2}}$. We showed in Case 1 that under this setting, we are better off serving type 1 casualties first and then type 2 casualties. In the second interval, we have $f_{2 t}^{s_{1}}>f_{1 t}^{s_{2}}$ as in Case 2, where we showed it is better to serving type 2 casualties first, and then type 1 casualties. Combining the results from these two intervals, we have a portion of type 1 casualties served first, then all type 2 casualties, and finally rest of the type 1 casualties.

Given the three possible cases, it is optimal not to interrupting service of type 2 casualties for a server.

PART 2: Without loss of generality, assume we have a partial schedule $S$ with two servers starting at time $t^{\prime}$, where server 1 serves two type 1 casualties and server 2 serves two type 2 . Same as PART 1, there are three cases in regard to where $t_{s}$ lies in regard to schedule $S$. For each of the three cases we show that schedule $S$ which is not balanced among servers in terms of service to difference casualty types, is suboptimal.

CASE 1: $\left(t_{s}>\max \left(2 s_{1}, 2 s_{2}\right)\right.$, thus $f_{2 t}^{\delta}<f_{1 t}^{\delta^{\prime}}, \forall t=t^{\prime}, t^{\prime}+s_{1}, t^{\prime}+s_{2}$ and $\left.\delta, \delta^{\prime}>0\right)$ : In this case, we switch the second type 1 casualty in server 1 with the first type 2 casualty in server 2 to form schedule $S^{\prime}$. The difference in total survival probabilities of schedules $S^{\prime}$ and $S$ is as follows:

$$
\begin{aligned}
T R\left(S^{\prime}\right)-T R(S) & =f_{1 t}+f_{2\left[t+s_{1}\right]}+f_{1 t}+f_{2\left[t+s_{1}\right]}-f_{1 t}-f_{1\left[t+s_{1}\right]}-f_{2 t}-f_{2\left[t+s_{2}\right]} \\
& =f_{2\left[t+s_{1}\right]}+f_{1 t}+f_{2\left[t+s_{1}\right]}-f_{1\left[t+s_{1}\right]}-f_{2 t}-f_{2\left[t+s_{2}\right]} \\
& =f_{2\left[t+s_{1}\right]}+f_{1 t}+f_{2\left[t+s_{1}\right]}-f_{1\left[t+s_{1}\right]}-f_{2 t}-f_{2\left[t+s_{2}\right]} \pm f_{1\left[t+s_{2}\right]} \\
& =\left(f_{1 t}^{s_{2}}-f_{2 t}^{s_{1}}\right)+\left(f_{1\left[t+s_{2}\right]}^{s_{1}-s_{2}}-f_{2\left[t+s_{2}\right]}^{s_{1}-s_{2}}\right)
\end{aligned}
$$


In this case, $f_{2 t}^{s_{2}}<f_{1 t}^{s_{1}}, \forall t=t^{\prime}, t^{\prime}+s_{1}, t^{\prime}+s_{2}$. Thus, both terms $\left(f_{1 t}^{s_{2}}-f_{2 t}^{s_{1}}\right)$ and $\left(f_{1\left[t+s_{2}\right]}^{s_{1}-s_{2}}-f_{2\left[t+s_{2}\right]}^{s_{1}-s_{2}}\right)$ are positive. As a result, $T R\left(S^{\prime}\right)-T R(S)>0$, which indicates unbalanced service among servers cannot be optimal.

CASE 2: $\left(t_{s}<t^{\prime}\right.$, thus $f_{1 t}^{\delta}<f_{2 t}^{\delta^{\prime}}, \forall t=t^{\prime}, t^{\prime}+s_{1}, t^{\prime}+s_{2}$ and $\left.\delta, \delta^{\prime}>0\right)$ :In this case, we switch the first type 1 casualty in server 1 with the second type 2 casualty in server 2 to form schedule $S^{\prime \prime}$. The difference in total survival probabilities of schedules $S^{\prime \prime}$ and $S$ is as follows:

$$
\begin{aligned}
T R\left(S^{\prime \prime}\right)-T R(S) & =f_{2 t}+f_{1\left[t+s_{2}\right]}+f_{2 t}+f_{1\left[t+s_{2}\right]}-f_{1 t}-f_{1\left[t+s_{1}\right]}-f_{2 t}-f_{2\left[t+s_{2}\right]} \\
& =f_{1\left[t+s_{2}\right]}+f_{2 t}+f_{1\left[t+s_{2}\right]}-f_{1 t}-f_{1\left[t+s_{1}\right]}-f_{2\left[t+s_{2}\right]} \\
& =f_{1\left[t+s_{2}\right]}+f_{2 t}+f_{1\left[t+s_{2}\right]}-f_{1 t}-f_{1\left[t+s_{1}\right]}-f_{2\left[t+s_{2}\right]} \pm f_{2\left[t+s_{1}\right]} \\
& =\left(f_{2 t}^{s_{1}}-f_{1 t}^{s_{2}}\right)+\left(f_{2\left[t+s_{2}\right]}^{s_{1}-s_{2}}-f_{1\left[t+s_{2}\right]}^{s_{1}-s_{2}}\right)
\end{aligned}
$$

Based on the assumption in this case $\left(f_{2 t}^{s_{2}}<f_{1 t}^{s_{1}}, \forall t=t^{\prime}, t^{\prime}+s_{1}, t^{\prime}+s_{2}\right)$, both terms above $\left(f_{2 t}^{s_{1}}-f_{1 t}^{s_{2}}\right)$ and $\left(f_{2\left[t+s_{2}\right]}^{s_{1}-s_{2}}-f_{1\left[t+s_{2}\right]}^{s_{1}-s_{2}}\right)$ are positive. Thus, we are better off balancing out service on both servers.

CASE 3: $\left(t^{\prime}<t_{s}<\max \left(2 s_{1}, 2 s_{2}\right)\right.$, thus $f_{1 t}^{\delta}>f_{2 t}^{\delta^{\prime}}, \forall t=t^{\prime}, \cdots, t_{s}$ and $\left.f_{1 t}^{\delta}<f_{2 t}^{\delta^{\prime}}, \forall t>t_{s}\right)$ : We break this case into two time intervals, $\left[t, t_{s}\right]$ and $\left[t_{s}, n_{1} s_{1}+n_{2} s_{2}\right]$. In the first interval we have $f_{2 t}^{s_{1}}<f_{1 t}^{s_{2}}$ similar to CASE 1, in which we showed that we are better off balancing service among two servers by arranging service to type 2 casualties towards the end. In the second interval $\left(\left[t_{s}, n_{1} s_{1}+n_{2} s_{2}\right]\right)$, we have $f_{1 t}^{\delta}<f_{2 t}^{\delta^{\prime}}, \forall t>t_{s}$ similar to CASE 2, in which we showed that we are better off balancing servers by arranging type 2 casualties at the beginning for each server. Combining the results from these two intervals, we have balanced servers serving type 2 casualties around (or as close as possible to) $t_{s}$.

The results from PART 2 can be extended to more than two servers by comparing two servers at a time. We can conclude that under Assumption 2, in the optimal solution to Model 1, service to type 2 casualties is not interrupted. 